\title{
Covid-19 e Tabagismo:Aspectos Epidemiológicos, Biológicos, Psicossociaise Implicações para a Política Nacional de Controle do Tabaco
}

doi: https://doi.org/10.32635/2176-9745.RBC.2020v66nTemaAtual.1039

COVID-19 and Smoking: Epidemiological, Biological, Psychosocial Aspects and Implications for the National Tobacco Control Policy

Covid-19 y Tabaquismo: Aspectos Epidemiológicos, Biológicos, Psicosociales e Implicaciones para la Política Nacional de Control del Tabaco

Tânia Maria Cavalcante'; Cristina de Abreu Perez²; Felipe Lacerda Mendes ${ }^{3}$; Alessandra Trindade Machado ${ }^{4}$; Erica Cavalcanti Rangel5; $^{5}$ Renata Cristina Arthou Pereira ${ }^{6}$; Rita de Cassia Martins7; Angela Machado de Miranda Leal ${ }^{8}$

\section{INTRODUÇÃO}

Segundo a Organização das Nações Unidas, a pandemia do novo coronavírus (2019-nCoV) é o maior desafio da humanidade desde a Segunda Guerra Mundial ${ }^{1}$. Os escassos conhecimentos sobre suas características epidemiológicas, biológicas, psicossociais, formas de prevenção e tratamento eficazes mobilizaram uma estratégia global sem precedentes, em uma corrida contra o tempo na busca de soluçôes para mitigar os impactos sanitários e socioeconômicos desse inimigo invisível. Um dos resultados desse aprendizado relaciona-se à identificação de grupos mais vulneráveis à transmissão e às complicaçôes de saúde pela doença pelo coronavírus 2019 (coronavirus disease 2019 - Covid-19), entre os quais, estão os fumantes, que, no mundo, representam cerca de 1 bilhão de pessoas e, no Brasil, mais de 20 milhôes ${ }^{2,3}$.

Importante lembrar que em 1986 a 39a Assembleia Mundial de Saúde reconheceu o tabagismo como uma pandemia ${ }^{4}$. Atualmente, essa pandemia responde por 8 milhôes de mortes anuais no mundo e impôe um custo global de 1,4 trilhão de dólares ao ano para todas as nações ${ }^{5,6}$. Já no Brasil, gera 157 mil mortes ao ano e um custo de mais de 57 bilhóes de reais ao ano ${ }^{7}$.
O objetivo deste artigo é reunir informaçôes sobre aspectos epidemiológicos, biológicos e psicossociais da relação entre tabagismo e Covid-19, colaborando com a reflexẫo sobre o potencial de medidas de prevenção e redução do tabagismo no rol de políticas públicas de combate ao novo coronavírus.

\section{DESENVOLVIMENTO}

\section{O TABAGISMO OFERECE UM RISCO MAIOR PARA A COVID-19?}

Crescem as evidências de que o tabagismo está associado a uma maior chance de resultados adversos na infecção pelo novo coronavírus. Entre os pacientes infectados, os fumantes têm um risco duas vezes maior de serem internados em unidade de terapia intensiva, de necessitar de ventilação mecânica e de virem a óbito do que pacientes não fumantes ${ }^{8-13}$

Os fumantes, quando comparados com não fumantes, apresentam um risco significativamente mais elevado de desenvolverem doenças crônicas, como câncer, cardiovasculares, doença pulmonar obstrutiva crônica e diabetes ${ }^{14}$. Eles também sofrem com efeitos adversos de caráter sistêmico, como alteração do funcionamento do sistema imune, o que explica o maior risco de infecçóes

${ }^{1}$ Médica. Doutora pelo Programa de Pós-Graduação Stricto Sensu em Oncologia do Instituto Nacional de Câncer José Alencar Gomes da Silva (INCA). Rio de Janeiro (RJ), Brasil. Orcid iD: https://orcid.org/0000-0003-0866-4672

2 Psicóloga. Mestre e Doutoranda em Saúde Pública pela Escola Nacional de Saúde Pública Sérgio Arouca. Fundação Oswaldo Cruz (Ensp/Fiocruz). INCA. Rio de Janeiro (RJ), Brasil. Orcid iD: https://orcid.org/0000-0002-5170-9664

${ }^{3}$ Bacharel em Direito. Mestrando em Saúde Pública pela Ensp/Fiocruz. INCA. Rio de Janeiro (RJ), Brasil. Orcid iD: https://orcid.org/0000-0003-1071-1597

${ }^{4}$ Cirurgiã-Dentista. Mestrado em Odontologia pela Universidade Federal de Minas Gerais (UFMG). INCA. Rio de Janeiro (RJ), Brasil. Orcid iD: https://orcid.org/ 0000-0003-3697-9217

${ }^{5}$ Assistente Social. Mestre em Saúde Pública pela Ensp/Fiocruz. INCA. Rio de Janeiro (RJ), Brasil. Orcid iD: https://orcid.org/0000-0001-8380-241x

${ }^{6}$ Enfermeira. Mestre em Saúde Pública pela Ensp/Fiocruz. INCA. Rio de Janeiro (RJ), Brasil. Orcid iD: https://orcid.org/0000-0002-3540-6305

7 Psicóloga. Pós-Graduada em Psicologia Clínica e Institucional pela Universidade Estácio de Sá. INCA. Rio de Janeiro (RJ), Brasil. Orcid iD: https://orcid.org/ 0000-0002-9965-0243

${ }^{8}$ Bacharel em Letras. Pós-Graduada em Gestão Pública pela Universidade Cândido Mendes. INCA. Rio de Janeiro (RJ), Brasil. Orcid iD: https://orcid.org/ 0000-0001-6752-8801

Endereço para correspondência: Cristina de Abreu Perez. Rua Pinheiro Guimarães, 150, apto. 503 - Botafogo. Rio de Janeiro (RJ), Brasil. CEP 22281-080. E-mail: cristinadeabreuperez@gmail.com 
respiratórias bacterianas e virais, e um maior risco de desenvolverem e morrerem por tuberculose, quando comparados com não fumantes ${ }^{14}$. A maioria dessas condiçóes e doenças tabaco-relacionadas foi identificada como fator de risco para as complicaçôes da Covid-19 15-20.

Quanto aos usuários de cigarros eletrônicos e de cigarros de tabaco aquecido, os estudos têm demonstrado que os vapores produzidos por esses dois produtos geram efeitos inflamatórios e tóxicos sobre os pulmóes e o sistema cardiovascular. Os Estados Unidos (EUA), um dos países onde mais cresceu o consumo de cigarros eletrônicos entre jovens, enfrentaram uma epidemia de casos graves de lesôes pulmonares agudas, acompanhados de insuficiência respiratória, em razão do uso desses produtos para vaporar, conhecidos como EVALI (do inglês, e-cigarette or vaping product use associaste lung injury ${ }^{21-24}$. Portanto, é plausível que o consumo desses dispositivos eletrônicos para fumar possa também contribuir para um elevado risco de complicaçôes pela Covid-19 entre seus usuários.

Alguns estudos, que identificaram uma baixa prevalência de fumantes entre pacientes hospitalizados com a Covid-19, quando comparada com a prevalência de fumantes na populaçáo em geral ${ }^{25,26}$, foram usados para especular sobre um possível papel protetor do tabagismo na infecção pelo coronavírus ${ }^{27-29}$. Esses estudos, contestados por diversas entidades de saúde, precisam ser interpretados com cautela, considerando a similaridade entre os mecanismos fisiopatológicos do tabagismo e da Covid-19 e a grande possibilidade de vieses de identificação do status de tabagismo entre os pacientes internados com essa infecção, o que pode erroneamente refletir em uma baixa prevalência de fumantes nesse grupo ${ }^{30-32}$.

\section{ASPECTOS DA TOXICIDADE DA FUMAÇA DE TABACO QUE TORNAM 0 FUMANTE MAIS VULNERÁVEL À GRAVIDADE DA INFECÇÃO POR COVID-19}

A fumaça gerada por cigarros e outros produtos de tabaco é um aerossol formado por mais de 7 mil substâncias químicas de diferentes classes ${ }^{33,34}$. Entre esses componentes, merece destaque a matéria particulada respirável, que funciona como importante agente inflamatório responsável por lesóes e trombos nos pulmôes e vasos sanguíneos ${ }^{35-39}$.

Já a nicotina, além de causar dependência química e atuar como agente promotor na carcinogênese, estimula a produção do peptídeo angiotensina II, que leva à vasoconstricção, o que, por sua vez, causa importante impacto sobre o sistema cardiovascular, especialmente no desenvolvimento de hipertensão arterial ${ }^{40-42}$.

Porém, entre um dos mais significativos elementos em comum da fisiopatologia da Covid-19 e do tabagismo, está a hipóxia. Observaçôes clínicas em pacientes infectados com Covid-19 descrevem um intrigante quadro de hipoxemia silenciosa grave, de caráter progressivo e pouco responsivo à ventilação mecânica ${ }^{16,43,44}$. Trata-se de um quadro de evolução rápida, que desencadeia um processo inflamatório agudo, com aumento do nível de fibrinogênio e D-dímeros, o qual tende a evoluir com coagulação intravascular disseminada, danos microvasculares trombóticos e uma tempestade de citocinas inflamatórias, resultando em maior permeabilidade vascular, falência múltipla de órgãos e morte ${ }^{45-49}$.

Entre fumantes, a hipóxia é crônica e resulta da inalaçáo de considerável volume de monóxido de carbono (CO) a cada tragada da fumaça de cigarros. A grande afinidade de ligação do $\mathrm{CO}$ com a hemoglobina gera carboxihemoglobina, prejudicando a oxigenação dos tecidos e órgãos e resultando em menor tolerância à atividade física e menor capacidade aeróbica ${ }^{50-56}$.

Potencializada pelo efeito de outras substâncias tóxicas da fumaça de tabaco, a hipóxia no fumante evolui com um mecanismo fisiopatológico similar ao observado na Covid-19, porém de evolução mais lenta, caracterizado por disfunção do endotélio dos vasos sanguíneos e por um processo inflamatório crônico, também mediado por citocinas ${ }^{57}$. Por sua vez, esse processo contribui para um estado de hipercoagulabilidade, caracterizado principalmente por aumento na agregação das plaquetas, aumento da produção de fibrinogênio pelo fígado e disfunçôes no processo de fibrinólise, importante mecanismo regulador do sistema hemostático que evita a formação ou remove os trombos intravasculares ${ }^{58-62}$. Esse desequilíbrio crônico nas funçóes de coagulação e anticoagulação entre fumantes explica, em parte, o risco aumentado de fenômenos tromboembólicos. Sob essa perspectiva, é plausível considerar que fumantes infectados pela Covid-19 tendem a ser mais vulneráveis à gravidade desse quadro por serem portadores de hipóxia crônica secundária ao tabagismo.

\section{ASPECTOS BIOLÓGICOS DO TABAGISMO QUE TORNAM FUMANTES MAIS VULNERÁVEIS À INVASÃO DAS CÉLULAS PELO NOVO CORONAVÍRUS}

Pesquisas que buscam explicar a relação entre a Covid-19 e o tabagismo apontam que o aumento na expressão da enzima de conversão da angiotensina 2 - ACE2 (do inglês, angiotensin-converting enzyme 2) entre fumantes seria um dos principais mecanismos biológicos responsáveis pelo maior risco de progressão e complicaçóes da Covid-19 nesse grupo. A ACE2 é uma proteína encontrada na membrana celular, na qual o vírus se acopla para invadir células e injetar seu material genético. Dentro da célula, o vírus passa a subverter o mecanismo de replicação celular em favor da replicação viral, processo seguido de destruição das células 
infectadas e expulsão dos virions (clones), os quais passam a invadir células vizinhas que tenham receptores ACE2. O sucessivo processo de invasões e replicaçōes será mais intenso quanto maior for a quantidade desses receptores nos órgãos previamente à sua infecção ${ }^{63-69}$.

Em situação normal, a ACE2 funciona como mediadora importante do sistema endócrino renina-angiotensina (SRA), presente nas membranas das células, especialmente das mucosas e de órgáos como coração, rins, pulmóes e trato gastrintestinal ${ }^{70}$. O SRA desempenha papel relevante na regulação da fisiologia cardiovascular e neurovascular ${ }^{40,71}$. De forma simplificada, o SRA conta com dois braços, que funcionam de forma antagônica, para manter o equilíbrio entre vasoconstricção e vasodilatação. Em um dos braços atua a enzima conversora de angiotensina (ACE), que transforma a angiotensina I em angiotensina II, tem ação vasoconstrictora potente e, quando liberada em excesso, contribui para a fisiopatogenia da hipertensão arterial. No outro braço, atua a ACE2, liberando angiotensina 1-7, que tem ação vasodilatadora importante para uma adequada função miocárdica e renal. Em resumo, essas duas enzimas (ACE e ACE2) atuam em uma espécie de gangorra nos dois braços do SRA para manter o equilíbrio entre vasoconstricção e vasodilatação na regulação das funçôes cardiovasculares, renais e neurovasculares ${ }^{70,72}$.

Também existem evidências de que o aumento da expressão de ACE2 representa uma resposta fisiológica protetiva frente a processos inflamatórios em vários tecidos, como os dos pulmóes, do fígado, do cérebro, além de desenvolver uma função protetora em condiçóes patológicas, como na aterosclerose, isquemia cerebral, obesidade, doença renal crônica e asma. Nessas situações, a função da ACE2 seria de inibir as citocinas, importantes agentes inflamatórios produzidos pelo sistema imunológico, e seus efeitos de proliferação, fibrose e hipertrofia nos tecidos ${ }^{73-75}$.

Entre fumantes, o aumento da expressão de ACE2 nas células pulmonares parece ser uma resposta protetiva dose-dependente do número de cigarros fumados por dia, frente aos processos inflamatórios desencadeados pela fumaça desse produto ${ }^{68}$, principalmente seu material particulado respirável de pequena dimensão, como os formados por partículas de até 2,5 micra (PM2,5) e o $\mathrm{CO}^{76-79}$. É plausível que essas alterações também ocorram entre usuários de cigarros eletrônicos ou cigarros de tabaco aquecido, em função da presença de PM2,5 no vapor que produzem ${ }^{80-82}$.

Vale destacar que a exposição ao PM2,5, também presente na poluição atmosférica, foi relacionada a uma maior taxa de mortalidade por coronavírus nos EUA e na Itália ${ }^{83-86}$.
Portanto, pode-se presumir que, se por um lado o aumento de ACE2 funciona como um mecanismo anti-inflamatório protetivo entre fumantes, por outro, ao funcionar como receptor para a entrada do vírus na célula, a ACE2 aumenta sua vulnerabilidade a uma maior carga viral na infecção pela Covid-19 ${ }^{87}$. Pode-se presumir ainda que, durante a evolução da infecção, a intensiva destruição de células com receptores ACE2 pelos vírus reduza o efeito protetor anti-inflamatório dessa enzima e contribua para a piora do quadro clínico $^{88}$. Estudos mais recentes têm chamado atenção para a tempestade de citocinas inflamatórias que ocorre durante a evoluçáo de quadros mais graves da Covid-19, fator crítico para falência múltipla de órgãos e óbito ${ }^{49,89,90}$.

Considerando esses aspectos, é possível que a presença de PM2,5 e CO na corrente secundária da fumaça de produtos de tabaco (a que polui ambientes internos) possa levar ao aumento da expressão da ACE2 nos pulmóes de fumantes passivos e também aumentar a vulnerabilidade desse grupo a uma maior carga viral e a chances de complicações da infecção pela Covid-19 $9^{69,91-93}$.

\section{O TABAGISMO PODE AUMENTAR O RISCO DE TRANSMISSÃO DA COVID-19?}

Além dos riscos de complicaçôes da Covid-19 entre fumantes, foi levantada a hipótese de que o ato de fumar em si poderia aumentar o risco de transmissão dessa infecção, pois envolve constante contato dos dedos com os lábios, caso as mãos tenham entrado em contato com alguma superfície infectada, incluindo o próprio cigarro. Da mesma forma, o uso de outros produtos para fumar que envolvem compartilhamento de bocais para inalar a fumaça, como cachimbo d'água ou narguilé, poderia também facilitar a transmissão do coronavírus entre seus usuários e para a comunidade ${ }^{94,95}$.

Estudos mostrando que o coronavírus se dissemina por meio de aerossóis nos quais podem permanecer viáveis por até 3 horas ${ }^{96-99}$ e a já conhecida relação entre infecções respiratórias sazonais e níveis de poluição atmosférica levaram à hipótese de que poluentes ambientais possam promover uma maior permanência de partículas do novo coronavírus no ar, ocasionando sua difusão indireta, além da sabida transmissão direta, pessoa a pessoa ${ }^{100-104}$. Sob essa perspectiva, é plausível que o risco da transmissão do coronavírus se aplique também aos fumantes passivos; ou seja, aos que respiram a fumaça ambiental de tabaco. Essa fumaça é um aerossol e inclui, entre outras substâncias tóxicas, o PM2,591-93,105, previamente mencionado, que pode funcionar como veículo para disseminação do vírus quando pessoas fumam em recintos coletivos contaminados ${ }^{106}$. 


\section{ASPECTOS PSICOSSOCIAIS DO TABAGISMO E IMPLICAÇ̃ES SOBRE} A COVID-19

No Brasil e em muitos outros países, a prevalência de fumantes tem se concentrado em grupos de menor renda e escolaridade ${ }^{3,107,108}$. As condiçōes de habitação desses grupos, em sua maioria, não permitem um distanciamento social adequado entre os membros da família, o que pode contribuir para que eles sejam mais afetados pela Covid-19 $19^{109-111}$.

É importante analisar, ainda, os efeitos psicossociais da pandemia sobre os índices nacionais de tabagismo, considerando o contexto de instabilidade econômica e psicológica, de isolamento social e as dificuldades de acesso à assistência hospitalar ${ }^{112,113}$. Estudos sobre o impacto da pandemia mostraram maior frequência de ansiedade, estresse e depressão associados ao medo da infecção pelo vírus. Tendo em vista que os fumantes parecem recorrer ao tabagismo para lidar com o estresse ${ }^{14}$, é importante considerar que todo esse cenário de confinamento, preocupação e incertezas durante a pandemia pode reforçar ainda mais a dependência da nicotina e contribuir para que fumantes fumem um número maior de cigarros em seus domicílios, aumentando a exposição de seus familiares à fumaça ambiental de tabaco e suas consequências, incluindo as relacionadas à transmissão da Covid-196,115.

\section{OS BENEFÍCIOS DA REDUÇÃO DO TABAGISMO NO ENFRENTAMENTO DA COVID-19}

Estudos mostram que o nível de expressão de ACE2 nas células, que tornam os fumantes mais vulneráveis à infeção pela Covid-19, tende a se normalizar entre ex-fumantes ${ }^{68}$.

Além disso, a cessaçáo de fumar melhora substantivamente outros aspectos fisiopatológicos decorrentes do tabagismo, que podem agravar as complicaçóes da Covid-19. A hipóxia, decorrente da intoxicaçấo crônica por $\mathrm{CO}$, tende a desaparecer depois das primeiras 8 horas de abstinência contínua ${ }^{53}$. Após um dia sem fumar, observa-se também a recuperação da disfunçáo do endotélio dos vasos e, depois de duas semanas de abstinência, a normalizaçăo da agregaçấo plaquetária e do nível de fibrinogênio no sangue, o que, por sua vez, reduz os riscos de eventos tromboembólicos e cardíacos entre fumantes ${ }^{62,116}$.

Esses achados tornam evidente a relevância de se investir em medidas para ampliar a cessação de fumar e prevenir a iniciação no tabagismo, como estratégias para reduzir os impactos da Covid-19 na população.

\section{CONCLUSÃO}

Assim como a Covid-19, o tabagismo também foi considerado uma pandemia pela Organização Mundial da Saúde (OMS), cujas respostas mais custo-efetivas para seu enfrentamento foram incluídas em um tratado internacional de saúde, a Convenção-Quadro da OMS para o Controle do Tabaco, em vigor há 15 anos. Apesar das evidências sobre a gravidade do tabagismo para a saúde pública, os esforços nacionais para o cumprimento integral desse tratado ainda sofrem retardos em função da interferência das grandes empresas transnacionais de tabaco.

Além de fator de risco para várias doenças crônicas graves, desde os anos 1990, o tabagismo é classificado como doença na $10^{a}$ Revisão do Código Internacional de Doenças da OMS e considerado uma doença pediátrica, pois quase a totalidade dos fumantes começa a fumar na adolescência, em decorrência de estratégias de marketing direcionadas a esse grupo. No entanto, apesar das políticas para seu controle, todos os dias, milhares de jovens ainda são induzidos a experimentar cigarros e começar a fumar.

Todas as evidências científicas tanto nos aspectos epidemiológicos, biológicos e psicossociais quanto do impacto negativo do tabagismo na transmissão e complicaçóes pela Covid-19 apontam que esse fator de risco contribuirá para ampliar a morbimortalidade pelo novo coronavírus e elevar os gastos do sistema de saúde com a compra de equipamentos, insumos e organização dos serviços.

Nesse sentido, políticas públicas para ampliar o acesso ao tratamento para cessação de fumar e medidas educativas e legislativas eficazes para a redução da iniciação de jovens no tabagismo precisam ser urgentemente priorizadas nas agendas estratégicas governamentais de combate à Covid-19 e outras pandemias futuras.

A vacina para conter a pandemia de Covid-19 ainda está em desenvolvimento. No entanto, o remédio para conter a pandemia do tabagismo já existe e depende da priorização dos interesses de saúde pública acima do lobby econômico das grandes corporaçóes de tabaco, garantindo a implementação plena da Convenção-Quadro da OMS para o Controle do Tabaco.

\section{CONTRIBUIÇÕES}

Tânia Maria Cavalcante contribuiu substancialmente na concepção do estudo; na obtenção, análise e interpretação dos dados; na redação e revisão crítica. Cristina de Abreu Perez e Felipe Lacerda Mendes contribuíram na obtenção, análise e interpretação dos dados; na redaçáo e revisão crítica. Alessandra Trindade Machado, Erica Cavalcanti Rangel, Renata Cristina Arthou Pereira, Rita de Cassia Martins e Angela Machado de Miranda Leal contribuíram na revisão crítica. Todos os autores aprovaram a versão final publicada. 


\section{DECLARAÇÃO DE CONFLITO DE INTERESSES}

Nada a declarar.

\section{FONTES DE FINANCIAMENTO}

Não há.

\section{REFERÊNCIAS}

1. Agência Brasil [Internet]. Brasília, DF: EBC; c2019. Pandemia é maior desafio desde a $2^{a}$ Guerra Mundial, alerta ONU; 2020 abr 04 [acesso 2020 abr 20]. Disponível em: https://agenciabrasil.ebc.com.br/ internacional/noticia/2020-04/pandemia-e-maiordesafio-desde-2a-guerra-mundial-alerta-onu

2. World Health Organization [Internet]. Geneva: WHO; c2020. Global Health Observatory (GHO) data: prevalence of tobacco smoking; [date unknown] [cited 2020 Apr 17]. Available from: https://www.who.int/ gho/tobacco/use/en/

3. Szklo AS, Souza MC, Szklo M, et al. Smokers in Brazil: who are they?. Tob Control. 2016 Sep;25(5):564-70. doi: https://doi.org/10.1136/tobaccocontrol-2015-052324

4. World Health Organization [Internet]. Geneva: WHO; c2020. WHA39.14 Tobacco or health; 1986 May 15 [cited 2020 Apr 17]. Available from: https://www.who. int/tobacco/framework/wha_eb/wha39_14/en/

5. World Health Organization [Internet]. Geneva: WHO; c2020. Tobacco: key facts; 2019 July 26 [cited 2020 Apr 17]. Available from: https://www.who.int/news-room/ fact-sheets/detail/tobacco

6. WHO Framework Convention on Tobacco Control [Internet]. Geneva: WHO; c2020. Press release, Tobacco kills more than 7 million people per year and is costing the world economy USD 1.4 trillion annually; 2017 May 30 [cited 2020 Apr 20]. Available from: https://www.who.int/fctc/mediacentre/press-release/ wntd-2017/en/

7. Marcia P, Bardach A, Palacios A, et al. Carga de doença atribuível ao uso do tabaco no Brasil e potencial impacto do aumento de preços por meio de impostos [Internet]. Buenos Aires: Instituto de Efectividad Clínica y Sanitaria; 2017 maio [acesso 2020 abr 20]. Disponível em: https://www.iecs.org.ar/wp-content/ uploads/Reporte-completo.pdf

8. Vardavas CI, Nikitara K. Covid-19 and smoking: a systematic review of the evidence. Tob Induc Dis. 2020;18:20. doi: https://doi.org/10.18332/tid/119324

9. Emami A, Javanmardi F, Pirbonyeh N, et al. Prevalence of underlying diseases in hospitalized patients with COVID-19: a systematic review and meta-analysis. Arch Acad Emerg Med [Internet]. 2020 [cited 2020 Apr 20];8(1):e35. Available from: https://www.ncbi.
nlm.nih.gov/pmc/articles/PMC7096724 Free PMC article. PMCID: PMC7096724.

10. Szklo AS. Associação entre fumar e progressão para complicações respiratórias graves em pacientes com Covid-19. Rev Bras Cancerol. 2020;66(2):e03974. doi: https://doi.org/10.32635/2176-9745. RBC.2020v66n2.974

11. Patanavanich R, Glantz SA. Smoking is associated with COVID-19 progression: a meta-analysis. MedRxiv [Preprint]. 2020 Apr 16. doi: https://doi.org/10.1101 /2020.04.13.20063669.

12. Mehra MR, Desai SS, Kuy SR, et al. Cardiovascular disease, drug therapy, and mortality in Covid-19. N Engl J Med. 2020 May 1. doi: https://doi.org/10.1056/ NEJMoa2007621

13. Zhou F, Yu T, Du R, et al. Clinical course and risk factors for mortality of adult inpatients with COVID-19 in Wuhan, China: a retrospective cohort study. Lancet. 2020 Mar 28;395(10229):1054-62. doi: https://doi.org/10.1016/S0140-6736(20)30566-3

14. Department of Health and Human Services (US). How tobacco smoke causes disease: the biology and behavioral basis for smoking-attributable disease: a report of the surgeon general [Internet]. Rockville, MD: Dept. of Health and Human Services (US), Public Health Service, Office of Surgeon General; 2010. [cited 2020 Apr 20]. Available from: http://www.ncbi.nlm. nih.gov/books/NBK53017

15. Centers for Disease Control and Prevention (US) [Internet]. Atlanta (GA): CDC; [date unknown]. Coronavirus disease 2019 (COVID-19): groups at higher risk for severe illness; [reviewed 2020 May 14; cited 2020 May 16]. Available from: https://www.cdc. gov/coronavirus/2019-ncov/need-extra-precautions/ groups-at-higher-risk.html

16. Pappas S. 'Silent hypoxia' may be killing COVID-19 patients. But there's hope. LiveScience [Internet]. 2020 Apr 23 [cited 2020 May 4]; News. Available from: https://www.livescience.com/silent-hypoxia-killingcovid-19-coronavirus-patients.html

17. Gattinoni L, Coppola S, Cressoni M, et al. Covid-19 does not lead to a "typical" acute respiratory distress syndrome. Am J Respir Crit Care Med. 2020;201(10):1299-1300. doi: https://doi.org/10.1164/rccm.202003-0817LE

18. Cui S, Chen S, Li X, et al. Prevalence of venous thromboembolism in patients with severe novel coronavirus pneumonia. J Thromb Haemost. $2020 \mathrm{Apr}$ 9. doi: https://doi.org/10.1111/jth.14830 Epub ahead of print. PubMed PMID: 32271988.

19. Arachchillage DRJ, Laffan M. Abnormal coagulation parameters are associated with poor prognosis in patients with novel coronavirus pneumonia. J Thromb Haemost. 2020;18(5):1233-4. doi: https://doi. org/10.1111/jth. 14820 
20. Liu Y, Bi L, Chen Y, et al. Active or latent tuberculosis increases susceptibility to COVID-19 and disease severity. MedRxiv [Preprint]. 2020 Mar 16. doi: https:// doi.org/10.1101/2020.03.10.20033795

21. American Lung Association [Internet]. Chicago: American Lung Association; [date unknown]. The impact of E-cigarette on the lungs; $2020 \mathrm{Feb} 24$ [cited 2020 Apr 27]. Available from: https://www.lung.org/ quit-smoking/e-cigarettes-vaping/impact-of-e-cigaretteson-lung

22. Chand HS, Muthumalage T, Maziak W, et al. Pulmonary toxicity and the pathophysiology of electronic cigarette, or vaping product, use associated lung injury. Front Pharmacol. 2019;10:1619. doi: https://doi.org/10.3389/fphar.2019.01619

23. Chun LF, Moazed F, Calfee CS, et al. Pulmonary toxicity of E-cigarettes. Am J Physiol Lung Cell Mol Physiol. 2017;313(2):L193-L206. doi: https://doi. org/10.1152/ajplung.00071.2017

24. MacDonald A, Middlekauff HR. Electronic cigarettes and cardiovascular health: what do we know so far? Vasc Health Risk Manag. 2019;15:159-74. doi: https://doi. org/10.2147/VHRM.S175970

25. Miyara M, Tubach F, Pourcher V. Low incidence of daily active tobacco smoking in patients with symptomatic COVID-19. Qeios [Preprint]. 2020 Apr 21. doi: https://doi.org/10.32388/WPP19W.3

26. Farsalinos K, Barbouni A, Niaura R. Smoking, vaping and hospitalization for COVID-19. Qeios [Preprint]. 2020 Mar 29. doi: https://doi.org/10.32388/ Z69O8A.13

27. Landim C. Fumar protege contra o COVID-19. 2020 abr 22 [acesso 2020 abr 27]. In: O Globo. Blog Ciência e Matemática [Internet]. Rio de Janeiro: O Globo. c1996 - 2020. Disponível em: https://blogs.oglobo. globo.com/ciencia-matematica/post/fumar-protegecontra-o-covid-19.html

28. UOL [Internet]. São Paulo: Universo Online; c1996 - 2020. [Video], Nicotina poderia ser arma contra Covid-19; 2020 abr 22 [acesso 2020 abr 27]; [1 min., 36 s]. Disponível em: https://noticias.uol.com.br/ videos/afp/2020/04/22/nicotina-poderia-ser-armacontra-covid-19.htm?cmpid=copiaecola

29. Os fumantes parecem estar mais protegidos contra o coronavírus. Por quê?. Exame [Internet]. 2020 maio 1 [acesso 2020 maio 4]; Ciência. Disponível em: https:// exame.abril.com.br/ciencia/os-fumantes-parecem-estarmais-protegidos-contra-o-coronavirus-por-que/

30. Instituto Nacional de Câncer José Alencar Gomes da Silva. Nota do INCA sobre estudo francês que levantou a hipótese de que a nicotina poderia ter papel protetor na COVID-19 [Internet]. 2020 abril [acesso 2020 maio 4]. Disponível em: https://www.inca.gov. $\mathrm{br} /$ sites/ufu.sti.inca.local/files//media/document/nota- inca_sobre_estudo_efeito_protetor-nicotina-covid-19_ versao_final_0.pdf

31. Shahab L, West R, Brown J. Review of: smoking, vaping and hospitalization for COVID-19. Qeios [Preprint]. 2020 Mar 27. doi: https://doi.org/10.32388/N5BBEF

32. ACT Promoção da Saúde; Associação Brasileira de Estudos do Álcool e outras Drogas; Associaçáo Médica Brasileira, et al. Nicotina e novo coronavírus: organizaçôes assinam nota conjunta; 2020 abril [acesso 2020 maio 8]. Disponível em: https://amb.org.br/ wp-content/uploads/2020/04/Nota_ACT_2404-1.pd f?fbclid=IwAR2 $w q c I I 0 S T C c n Q K 5 C 5 h 0 y g P X S i E E L P$ Vx-qUJSRgOKOFi9JLUVWBITf_yfc

33. Food and Drug Administration (US) [Internet]. Silver Spring (MD): Food and Drug Administration (US); [date unknown]. [Video], Chemicals in cigarettes: from plant to product to puff; 2019 May 16 [cited 2020 May 18]; [3 min., $30 \mathrm{sec}$. Available from: https:// www.fda.gov/tobacco-products/products-ingredientscomponents/chemicals-cigarettes-plant-product-puff

34. Centers for Disease Control and Prevention (US) [Internet]. Atlanta (GA): CDC; [date unknown]. Secondhand Smoke (SHS) Facts; [reviewed 2018 Jan 17; cited 2020 May 8]. Available from: https:// www.cdc.gov/tobacco/data_statistics/fact_sheets/ secondhand_smoke/general_facts/index.htm

35. Chen SY, Chan CC, Su TC. Particulate and gaseous pollutants on inflammation, thrombosis, and autonomic imbalance in subjects at risk for cardiovascular disease. Environ Pollut. 2017 Apr;223:403-8. doi: https://doi. org/10.1016/j.envpol.2017.01.037

36. Wu W, Jin Y, Carlsten C. Inflammatory health effects of indoor and outdoor particulate matter. J Allergy Clin Immunol. 2018 Mar;141(3):833-844. doi: https://doi. org/10.1016/j.jaci.2017.12.981

37. Ostro B, Malig B, Broadwin R, et al. Chronic PM2.5 exposure and inflammation: determining sensitive subgroups in mid-life women. Environ Res. 2014 Jul;132:168-175. doi: https://doi.org/10.1016/j. envres.2014.03.042

38. Environmental Protection Agency (US). Washington: EPA; [date unknown]. Health and environmental effects of particulate matter (PM); [last updated 2020 Apr 13; cited 2020 May 7]. Available from: https:// www.epa.gov/pm-pollution/health-and-environmentaleffects-particulate-matter-pm

39. Strzelak A, Ratajczak A, Adamiec A, et al. Tobacco smoke induces and alters immune responses in the lung triggering inflammation, allergy, asthma and other lung diseases: a mechanistic review. Int J Environ Res Public Health. 2018 May;15(5):1033. doi: https://doi. org/10.3390/ijerph 15051033

40. Oakes JM, Fuchs RM, Gardner JD, et al. Nicotine and the Renin-Angiotensin System. Am J Physiol Regul 
Integr Comp Physiol. 2018 Nov 1;315(5): R895-R906. doi: https://doi.org/10.1152/ajpregu.00099.2018

41. Yuan YM, Luo L, Guo Z, et al. Activation of Renin-Angiotensin-Aldosterone System (RAAS) in the lung of smoking-induced pulmonary arterial hypertension (PAH) rats. J Renin Angiotensin Aldosterone Syst. 2015;16(2):249-53. doi: https://doi. org/10.1177/1470320315576256

42. Olds JL, Kabbani N. Is nicotine exposure linked to cardiopulmonary vulnerability to COVID-19 in the general population?. FEBS J. 2020 Mar 18. doi: https:// doi.org/10.1111/febs.15303

43. Medscape [Internet]. New York: WebMD; c1994-2020. [Video], Ferenchick GS, Ferenchick HRB. 'Silent Hypoxemia' and other curious clinical observations in COVID-19; 2020 Apr 16 [cited 2020 May 6]; [4 min., 25 sec]. Available from: https://www.medscape. com/viewarticle/928803

44. Ottestad W, Seim M, Mæhlen JO. COVID-19 with silent hypoxemia. Tidsskr Nor Laegeforen. 2020 Apr 11;140(7). doi: https://doi.org/10.4045/ tidsskr.20.0299

45. American Society of Hematology [Internet]. Washington: ASH; c2020. COVID-19 and coagulopathy: frequently asked questions; [updated 2020 Apr 14; cited 2020 May 8]. Available from: https://www.hematology.org/ covid-19/covid-19-and-coagulopathy

46. Tang N, Bai H, Chen X, et al. Anticoagulant treatment is associated with decreased mortality in severe coronavirus disease 2019 patients with coagulopathy. J Thromb Haemost. 2020;18(5):1094-9. doi: https:// doi.org/10.1111/jth.14817

47. Magro C, Mulvey JJ, Berlin D, et al. Complement associated microvascular injury and thrombosis in the pathogenesis of severe COVID-19 infection: a report of five cases. Transl Res. 2020 Apr 15;S1931-5244(20)300700. doi: https://doi.org/10.1016/j.trsl.2020.04.007 Epub ahead of print. PubMed PMID: 32299776.

48. Jose RJ, Manuel A. COVID-19 cytokine storm: the interplay between inflammation and coagulation. Lancet Respir Med. 2020 April 2. doi: https://doi. org/10.1016/S2213-2600(20)30216-2

49. Zhang C, Wu Z, Li JW, et al. The cytokine release syndrome (CRS) of severe COVID-19 and Interleukin-6 receptor (IL-6R) antagonist Tocilizumab may be the key to reduce the mortality. Int J Antimicrob Agents. 2020 Mar 29. doi: https://doi.org/10.1016/j. ijantimicag.2020.105954 Epub ahead of print. PubMed PMID: 32234467.

50. Fricker M, Goggins BJ, Mateer S, et al. Chronic cigarette smoke exposure induces systemic hypoxia that drives intestinal dysfunction. JCI Insight. 2018 Feb 8;3(3):e94040. doi: https://doi.org/10.1172/jci. insight. 94040
51. Astrup P. Carbon Monoxide, smoking, and cardiovascular disease. Circulation. 1973 Dec;48(6):1167-8. doi: https://doi.org/10.1161/01.CIR.48.6.1167

52. Duran C, Winnicka L, Subramanian CR, et al. Recurrent carbon monoxide poisoning due to smoking leading to cognitive decline. Chest J. 2016;150(4 Suppl):423A. doi: https://doi.org/10.1016/j.chest.2016.08.436

53. Sandberg A, Sköld CM, Grunewald J, et al. Assessing recent smoking status by measuring exhaled carbon monoxide levels. PLoS One. 2011;6(12):e28864. doi: https://doi.org/10.1371/journal.pone.0028864

54. Milman N, Pedersen AN. Blood haemoglobin concentrations are higher in smokers and heavy alcohol consumers than in non-smokers and abstainers: should we adjust the reference range?. Ann Hematol. 2009;88(7):687-94. doi: https://doi.org/10.1007/ s00277-008-0647-9

55. Papathanasiou G, Mamali A, Papafloratos S, et al. Effects of smoking on cardiovascular function: the role of nicotine and carbon monoxide. Health Sci J [Internet]. 2014 [cited 2020 May 8];8(2):274-90. Available from: https://www.hsj.gr/medicine/effectsof-smoking-on-cardiovascular-function-the-role-ofnicotine-and-carbon-monoxide.pdf

56. Medscape [Internet]. New York: WebMD; c19942020. Nagalla S. Secondary polycythemia clinical presentation; 2018 Dec 20 [cited 2020 May 6]. Available from: https://emedicine.medscape.com/ article/205039-clinical\#b5

57. Barbieri SS, Zacchi E, Amadio P, et al. Cytokines present in smokers' serum interact with smoke components to enhance endothelial dysfunction. Cardiovascular Research. 2011;90(3):475-83. doi: https://doi. org/10.1093/cvr/cvr032

58. Pamukcu B, Oflaz H, Onur I. Effect of cigarette smoking on platelet aggregation. Clin Appl Thromb Hemost. 2011;17(6):E175-E180. doi: https://doi. org/10.1177/1076029610394440

59. Barua RS, Sy F, Srikanth S, et al. Effects of cigarette smoke exposure on clot dynamics and fibrin structure. Arterioscler Thromb Vasc Biol. 2010;30(1):75-79. doi: https://doi.org/10.1161/ATVBAHA.109.195024

60. Nielsen VG, Hafner DT, Steinbrenner EB. Tobacco smoke-induced hypercoagulation in human plasma: role of carbon monoxide. Blood Coagul Fibrinolysis. 2013;24(4):405-10. doi: https://doi.org/10.1097/ MBC.0b013e32835d5458

61. Pretorius E, Oberholzer HM, van der Spuy WJ, et al. Smoking and coagulation: the sticky fibrin phenomenon. Ultrastructural Pathology. 2010;34(4):236-9. doi: https://doi.org/10.3109/01913121003743716

62. Tapson VF. The role of smoking in coagulation and thromboembolism in chronic obstructive pulmonary disease. Proc Am Thorac Soc. 2004;2(1):71-7. doi: https://doi.org/10.1513/pats.200407-038MS 
63. Cai G, Bossé Y, Xiao F, et al. Tobacco smoking increases the lung gene expression of ACE2, the receptor of SARS-CoV-2. Am J Respir Crit Care Med. 2020 Apr 24. doi: https://doi.org/10.1164/rccm.202003-0693LE Epub ahead of print. PubMed PMID: 32329629.

64. Berlin I, Thomas D, Le Faou AL. COVID-19 and smoking. Nicotine Tob Res. 2020 Apr 3. doi: https:// doi.org/10.1093/ntr/ntaa059

65. Kearney J. Chloroquine as a potential treatment and prevention measure for the 2019 novel coronavirus: a review. Preprints. 2020 Mar 17. doi: https://doi. org/10.20944/preprints202003.0275.v1

66. Wang J, Luo Q, Chen R, et al. Susceptibility analysis of COVID-19 in smokers based on ACE2. Preprints. 2020 Mar 5. doi: https://doi.org/10.20944/ preprints202003.0078.v1

67. Leung JM, Yang CX, Tam A, et al. ACE-2 expression in the small airway epithelia of smokers and COPD patients: implications for COVID-19. Eur Respir J. 2020 May 14;55(5):2000688. doi: https://doi. org/10.1183/13993003.00688-2020

68. Smith JC, Sheltzer JM. Cigarette smoke triggers the expansion of a subpopulation of respiratory epithelial cells that express the SARS-CoV-2 receptor ACE2. BioRxiv [Preprint]. 2020 Mar 31. doi: https://doi. org/10.1101/2020.03.28.013672

69. University of California Merced [Internet]. Merced (CA): c2020. Morgante M. COVID-19 more likely to impact smokers and people exposed to secondhand smoke, report finds; 2020 Apr 2 [cited 2020 May 6]. Available from: https://news.ucmerced.edu/news/2020/ covid-19-more-likely-impact-smokers-and-peopleexposed-secondhand-smoke-report-finds

70. Burrell LM, Johnston CI, Tikellis C, et al. ACE2, a new regulator of the renin-angiotensin system. Trends Endocrinol Metab. 2004 May;15(4):166-9. doi: https:// doi.org/10.1016/j.tem.2004.03.001

71. Oudit GY, Crackower MA, Backx PH, et al. The role of ACE2 in cardiovascular physiology. Trends Cardiovasc Med. 2003 Apr;13(3):93-101. doi: https:// doi.org/10.1016/s1050-1738(02)00233-5

72. Mukerjee $S$, Gao $\mathrm{H}, \mathrm{Xu}$ J, et al. ACE2 and ADAM17 interaction regulates the activity of presympathetic neurons. Hypertension. 2019 Nov;74(5):1181-91. doi: https://doi.org/10.1161/ HYPERTENSIONAHA.119.13133

73. Chiusano ML. The modelling of COVID19 pathways sheds light on mechanisms, opportunities and on controversial interpretations of medical treatments. v2. arXiv 2003.11614 [Preprint]. 2020 Mar 25 [cited 2020 Apr 22]: [15 p.]. Available from: https://arxiv.org/ftp/ arxiv/papers/2003/2003.11614.pdf

74. Gaddam RR, Chambers S, Bhatia M. ACE and ACE2 in inflammation: a tale of two enzymes. Inflamm
Allergy Drug Targets. 2014;13(4):224-34. doi: https:// doi.org/10.2174/1871528113666140713164506

75. Chen J, Zhao Y, Chen S, et al. Neuronal over-expression of ACE2 protects brain from ischemia-induced damage. Neuropharmacology. 2014 Apr;79:550-8. doi: https:// doi.org/10.1016/j.neuropharm.2014.01.004

76. Lin CI, Tsai CH, Sun YL, et al. Instillation of particulate matter 2.5 induced acute lung injury and attenuated the injury recovery in ACE2 knockout mice. Int J Biol Sci. 2018;14(3):253-65. doi: https://doi.org/10.7150/ ijbs.23489

77. Lin CI, Wang GH, Cho CC, et al. Disorder of ReninAngiotensin system promoting the acute pulmonary inflammation by particulate matter $2.5\left(\mathrm{PM}_{2.5}\right)$ Exposure. FASEB J [Internet]. 2017 [cited 2020 Apr 22];31(1 Suppl). Available from: https://www.fasebj. org/doi/abs/10.1096/fasebj.31.1_supplement.854.6

78. Aztatzi-Aguilar OG, Uribe-Ramírez M, Arias-Ontaño JA, et al. Acute and subchronic exposure to air particulate matter induces expression of angiotensin and bradykinin-related genes in the lungs and heart: Angiotensin-II type-I receptor as a molecular target of particulate matter exposure. Part Fibre Toxicol. 2015;12:17. doi: https://doi.org/10.1186/s12989-0150094-4

79. Joshi S, Wollenzien H, Leclerc E, et al. Hypoxic regulation of angiotensin-converting enzyme 2 and Mas receptor in human $\mathrm{CD}^{4} 4^{+}$Cells. J Cell Physiol. 2019 Nov;234(11):20420-31. doi: https://doi.org/10.1002/ jcp. 28643

80. Fernández E, Ballbè M, Sureda X, et al. Particulate matter from electronic cigarettes and conventional cigarettes: a systematic review and observational study. Curr Environ Health Rep. 2015 Dec;2(4):423-9. doi: https://doi.org/10.1007/s40572-015-0072-x

81. Qasim H, Karim ZA, Rivera JO, et al. Impact of electronic cigarettes on the cardiovascular system. J Am Heart Assoc. 2017;6(9). doi: https://doi.org/10.1161/ JAHA.117.006353

82. Ruprecht AA, De Marco C, Saffari A, et al. Environmental pollution and emission factors of electronic cigarettes, heat-not-burn tobacco products, and conventional cigarettes. Aerosol Sci Technol. 2017;51(6):674-84. doi: https://doi.org/10.1080/02786826.2017.13002 31

83. Havard School of Public Health [Internet]. Boston: The President and Fellows of Harvard College; c1999-2020. Air pollution linked with higher COVID-19 death rates; [updated 2020 May 5; cited 2020 May 8]. Available from: https://www.hsph.harvard.edu/news/hsph-in-thenews/air-pollution-linked-with-higher-covid-19-deathrates/

84. Conticini E, Frediani B, Caro D. Can atmospheric pollution be considered a co-factor in extremely high level of SARS-CoV-2 lethality in Northern Italy? 
Environ Pollut. 2020 Jun;261:114465. doi: https:// doi.org/10.1016/j.envpol.2020.114465

85. Deutsche Welle [Internet]. Germany: DW; c2020. Niranjan A. Coronavirus: air pollution might raise risk of fatality; 2020 Apr 07 [cited 2020 Apr 22]. Available from: https:/www.dw.com/en/coronavirusair-pollution-might-raise-risk-of-fatality/a-52977422

86. Aarhus University. Link between air pollution and coronavirus mortality in Italy could be possible. Science Daily [Internet]. 2020 Apr 6 [cited 2020 Apr 22];Science News. Available from: https://www. sciencedaily.com/releases/2020/04/200406100824. htm

87. Huang JGZ, Lin L, Lv J. Coronavirus disease 2019 (COVID-19) and cardiovascular disease: a viewpoint on the potential influence of angiotensin-converting enzyme inhibitors/Angiotensin receptor blockers on onset and severity of severe acute respiratory syndrome coronavirus 2 infection. J Am Heart Assoc. 2020 Apr 7;9(7):e016219. doi: https://doi.org/10.1161/ JAHA.120.016219.

88. American Collee of Cardiology [Internet]. Washington: American College of Cardiology; c2020. Foundation. Mukherjee D. COVID-19 and Renin Angiotensin blockers; 2020 Apr 17 [cited 2020 May 3]. Available from: https://www.acc.org/latest-in-cardiology/tenpoints-to-remember/2020/04/17/13/16/covid-19-andrenin-angiotensin-blockers

89. George A. Cytokine storm: an overreaction of the body's immune system. NewScientist [Internet]. [date unknown] [cited 2020 May 8]. Available from: https:// www.newscientist.com/term/cytokine-storm/

90. MehtaP,McAuleyDF,Brown M, etal.COVID-19: consider cytokine storm syndromes and immunosuppression. Lancet. 2020 Mar 28;395(10229):1033-4. doi: https:// doi.org/10.1016/S0140-6736(20)30628-0

91. Loffredo CA, Tang Y, Momen $\mathrm{M}$, et al. $\mathrm{PM}_{2.5}$ as a marker of exposure to tobacco smoke and other sources of particulate matter in Cairo, Egypt. Int J Tuberc Lung Dis. 2016 Mar;20(3):417-22. doi: https://doi. org/10.5588/ijtld.15.0316

92. Kaplan B, Carkoglu A, Ergor G, et al. evaluation of secondhand smoke using PM2.5 and observations in a random stratified sample in hospitality venues from 12 cities. Int J Environ Res Public Health. 2019 Apr 17;16(8):1381. doi: https://doi.org/10.3390/ ijerph16081381

93. Nafees AA, Taj T, Kadir MM, et al. Indoor air pollution $\left(\mathrm{PM}_{2.5}\right)$ due to secondhand smoke in selected hospitality and entertainment venues of Karachi, Pakistan. Tob Control. 2012 Sep;21(5):460-4. doi: https://doi. org/10.1136/tc.2011.043190

94. World Health Organization [Internet]. Geneva: WHO; c2020. Q\&A: Tobacco and COVID-19; 2020 May
24 [cited 2020 May 7]. Available from: https://www. who.int/news-room/q-a-detail/q-a-on-smoking-andcovid-19

95. WHO Framework Convention on Tobacco Control [Internet]. Beirut: FCTC; c2019. Najen J. Increased risk of COVID-19 infection amongst smokers and amongst waterpipe users; [cited 2020 May 8]. Available from: https://untobaccocontrol.org/kh/waterpipes/ covid-19/

96. Doremalen N, Morris DH, Holbrook MG, et al. Aerosol and surface stability of SARS-CoV-2 as compared with SARS-CoV-1. N Engl J Med. 2020 Apr 16;382(16):1564-1567. doi: https://doi.org/10.1056/ NEJMc2004973

97. Young J. Japanese scientists find new coronavirus transmission route in breakthrough study. CCN [Internet]. 2020 Mar 30 [cited 2020 May 8]; Headlines. Available from: https:/www.ccn.com/japan-scientistsfind-new-transmission-route-of-coronavirus-inbreakthrough-study

98. Cientistas acham coronavírus em amostras de ar distantes até 4 metros de doentes. CCIH [Internet]. 2020 abr 13 [acesso 2020 maio 8];COVID-19 (coronavírus): atualizaçôes. Disponível em: https:// www.ccih.med.br/covid-19-13-abril/

99. Gorbunov B. Aerosol particles laden with COVID-19 travel over $30 \mathrm{~m}$ distance. Preprints. 2020;2020040546. doi: https://doi.org/10.20944/ preprints202004.0546.v1

100. Su W, Wu X, Geng X, et al. The short-term effects of air pollutants on influenza-like illness in Jinan, China. BMC Public Health. 2019;19:1319. doi: https:/doi. org/10.1186/s12889-019-7607-2

101. Frontera A, Martin C, Vlachos K, et al. Regional air pollution persistence links to COVID-19 infection zoning [letter]. J Infect. 2020 Apr 10. doi: https://doi. org/10.1016/j.jinf.2020.03.045

102. Forthomme C. COVID-19: link with air pollution? Italy's and China's experience. Impakter [Internet]. 2020 Mar 27 [update 2020 Apr 9; cited 2020 May 9]. Available from: https://impakter.com/covid-19-linkwith-air-pollution-italys-and-chinas-experience/

103. Setti L, Passarini F, De Gennaro G, et al. SARSCov-2 RNA found on particulate matter of Bergamo in northern Italy: first preliminary evidence. MedRxiv [Preprint]. 2020 Apr 24. doi: https://doi. org/10.1101/2020.04.15.20065995

104. Setti L. Is there a plausible role for particulate matter in the spreading of COVID-19 in northern Italy?. BMJ [Internet] 2020 Apr 8. [cited 2020 May 15]. Available from: https://www.bmj.com/content/368/ bmj.m1103/rr

105. eCycle [Internet]. [sem local]: eCycle; 2010-2020. Aires L. Os perigos do material particulado [acesso 2020 maio 
10]. Disponível em: https://www.ecycle.com.br/1379material-particulado

106. Puleo M. How does the new coronavirus spread? Think of second-hand smoke. Acuuweather [Internet]. 2020 Mar 23 [update 2020 May 14; cited 2020 May 15];News/health. Available from: https://www. accuweather.com/en/health-wellness/how-doesthe-new-coronavirus-spread-think-of-second-handsmoke/706489

107. World Health Organization [Internet]. Geneva: WHO; c2020. Tobacco: key facts; 2019 Jul 26 [cited 2020 May 15]. Available from: https://www.who.int/news-room/ fact-sheets/detail/tobacco

108. World Health Organization. Tobacco and poverty: a vicious circle [Internet]. Geneva: WHO; 2004 [cited 2020 May 15]. Available from: https:// apps.who.int/iris/bitstream/handle/10665/116428/ EMRPUB_2004_EN_773.pdf

109. Wilson C. These graphs show how covid-19 is ravaging new york city's low-income neighborhoods. Time [Internet]. 2020 Apr 15 [cited 2020 May 15]; Health Covid-19. Available from: https://time.com/5821212/ coronavirus-low-income-communities/

110. Mahler DG, Laknerr C, Aguilar RAC, et al. The impact of COVID-19 (Coronavirus) on global poverty: why Sub-Saharan Africa might be the region hardest hit. 2020 Apr 20 [cited 2020 May 14]. In: World Bank. Data Blog [Internet]. Washington: The World Bank Group. c2020. Available from: https://blogs.worldbank.org/opendata/ impact-covid-19-coronavirus-global-poverty-why-subsaharan-africa-might-be-region-hardest

111. Arantes JT. Desigualdade social torna o combate à COVID-19 ainda mais difícil. Agência FAPESP [Internet]. 2020 abr 16 [acesso 2020 maio 15]. Disponível em: http://agencia.fapesp.br/desigualdadesocial-torna-o-combate-a-covid-19-ainda-maisdificil/32969/

112. Volkow ND. Collision of the COVID-19 and addiction epidemics. Ann Intern Med. 2020 Apr 2;M20-1212. doi: https://doi.org/10.7326/M20-1212 Epub ahead of print. PubMed PMID: 32240293.

113. Patwardhan P. COVID-19: risk of increase in smoking rates among England's 6 million smokers and relapse among England's 11 million ex-smokers. BJGP Open. 2020 Apr 7;bjgpopen20X101067. doi: https://doi. org/10.3399/bjgpopen20X101067 Epub ahead of print. PubMed PMID: 32265183.

114. Meule A, Reichenberger J, Blechert J. Smoking, stress eating, and body weight: the moderating role of perceived stress. Subst Use Misuse. 2018 Nov 10;53(13):2152-2156. doi: https://doi.org/10.1080/1 0826084.2018.1461223

115. Simons D, Perski O, Brown J. Covid-19: the role of smoking cessation during respiratory virus epidemics.
BMJ Opinion [Internet]. 2020 Mar 20 [cited 2020 May 08]. Available from: https://blogs.bmj.com/ bmj/2020/03/20/covid-19-the-role-of-smokingcessation-during-respiratory-virus-epidemics/

116. Morita H, Ikeda H, Haramaki N, et al. Only two-week smoking cessation improves platelet aggregability and intraplatelet redox imbalance of long-term smokers. J Am Coll Cardiol. 2005 Feb 15;45(4):589-94. doi: https://doi.org/10.1016/j.jacc.2004.10.061

Recebido em 17/5/2020 Aprovado em 20/5/2020 